\title{
LA COORDINACIÓN GUBERNAMENTAL DE LAS POLÍTICAS EUROPEAS. UN ANÁLISIS DE DERECHO COMPARADO SOBRE LOS MIEMBROS FUNDADORES
}

\author{
Sabrina Ragone
}

\begin{abstract}
Sumario: 1. Introducción. 2. Metodología: un estudio de DereCHO CONSTITUCiOnal COMPARAdO y Europeo. 3. SOluciones COMPARADAS: UNA OJEADA RÁPIDA. 4. LA COORDINACIÓN GUBERNAMENTAL. 4.1. Modelo 1: la opción cero, es decir, la coordinación sin Ministro o sede específica. 4.2. Modelo 2: la coordinación sin Ministro, pero con una sede en el Ministerio de Asuntos Exteriores. 4.3. Modelo 3: la coordinación sin Ministro, pero con una sede en la Presidencia del Gobierno. 4.4. Modelo 4: la coordinación con Ministro y con una sede en la Presidencia del Gobierno. 5. CONCLUSIONES.
\end{abstract}

\section{INTRODUCCIÓN}

¿Con qué mecanismos las Administraciones nacionales gestionan de forma coordinada y/o conjunta las políticas europeas? En particular, ¿qué instrumentos prevén los ordenamientos jurídicos de los Estados miembros para que los distintos Ministerios afectados se coordinen? ¿Qué modelos de referencia es posible elaborar a raíz de las experiencias concretas de los países fundadores?

A estas cuestiones se intentará contestar a lo largo del presente trabajo, que tiene como punto de partida un dato evidente: la afectación de los sistemas normativos estatales por parte de las fuentes de la $\mathrm{UE}^{1}$ y la «europeiza-

${ }^{1}$ Lo demuestra el hecho de que algunos países hayan aprobado reformas constitucionales para dar un reconocimiento explícito a los avances de la Unión y a su participación en ella. Entre las Constituciones de los Estados fundadores, se puede mencionar el art. 23 de la alemana, el art. 88-1 hasta el 88-7 de la francesa o el art. 117 de la italiana (con referencia al ejercicio de las competencias legislativas). 
ción» de los Estados miembros ${ }^{2}$ declinada también con referencia a la vertiente institucional, que es la que nos interesa.

$\mathrm{Al}$ respecto, se puede hacer referencia a estudios empíricos, conceptuales y teóricos ${ }^{3}$, en los cuales se evidencia que no se ha dado una convergencia sustancial entre los Estados miembros. Y ello a pesar de los factores que podrían llevar a la adopción de soluciones parecidas -cuando no idénticas-, como el contexto normativo y las dinámicas comunes a nivel europeo ${ }^{4}$. Sin embargo, conviven, en la UE, sistemas heterogéneos de coordinación, debido probablemente a factores que se sitúan básicamente en el nivel nacional (pero sobre este punto volveremos más adelante). Entre ellos, se va a mencionar, en primer lugar, la actitud del Estado hacia la integración europea (proactiva, reactiva, pasiva...); en segundo lugar, la importancia de los asuntos europeos en la política interna (a efectos electorales y/o de coalición con otros partidos) y la sensibilidad específica sobre determinados temas (como las políticas de agricultura, pesca...); en tercer lugar, el apoyo de la sociedad civil a la pertenencia a la Unión; en cuarto lugar, la tendencia a consultar distintos actores a nivel interno y la concepción de la coordinación (como manifestación del poder de dirección del Presidente u ocasión de diálogo y confrontación efectiva); finalmente, el modelo de Administración y la existencia de ejecutivos regionales o estatales ${ }^{5}$.

Este proceso de adaptación institucional ${ }^{6}$ como consecuencia de los cambios supranacionales ha afectado a varios órganos constitucionales,

${ }^{2}$ Véanse sobre este fenómeno DebBasch, CH. (ed.), Administrations nationales et intégration européenne, Centre de Recherches Administratives, Aix-Marseille, 1987; MÉny, Y., Muller, P. y Quermonne, J.L. (eds.), Adjusting to Europe. The Impact of the European Union on national institution and policies, Routledge, Londres, 1996; GoETz, K.H. y HIx, S., Europeanised Politics? European Integration and National Political Systems, Frank Cass, Londres, 2000; GraZiano, P. y VInK, M. (eds.), Europeanization: New Research Agendas, Palgrave, Basingstoke, 2007. Interesante la distinción entre europeización vertical y horizontal realizada por MAGONE, J.M., Contemporary European Politics. A Comparative Introduction, Routledge, Abingdon-Nueva York, 2011, p. 158 y ss.

${ }^{3}$ Como lo subrayan Goetz, K.H. y MeYer-SAhling, J.H., «The Europeanisation of national political systems: Parliaments and executives», Living Reviews in European Governance, Vol. 3, n. 2, 2008, p. 4.

${ }^{4}$ Sobre los incentivos europeos a la adopción de un buen sistema de gobernanza, con especial referencia a algunos de los países que estaban a punto de entrar en la Unión, véase LIPPERT, B., UMBACH, G. y WESSELS. W., «Europeanization of CEE executives: EU membership negotiations as a shaping power», Journal of European Public Policy, Vol. 8, n. 6, 2001, p. 984 y ss.

5 Véase al respecto el examen de Kassim, H., Peters, B.G. y Wright, V. (eds.), The National Co-ordination of EU Policy. The Domestic Level, Oxford University Press, Oxford, 2000, p. 10 y ss.

${ }^{6}$ Una definición eficaz de este proceso se encuentra en OLSEN, J.P., «European Challenge to the Nation State», en Steunenberg, B. y van Vught, F. (eds.), Political Institu- 
pero el que se ha visto mayormente afectado, desde el principio, es el Gobierno ${ }^{7}$.

Los análisis (especialmente politológicos) relativos a la influencia de la UE sobre los ejecutivos nacionales han constatado dos efectos: en primer lugar, la modificación de los equilibrios institucionales internos, pues la integración ha ido consolidando el papel de los Gobiernos nacionales, que fueron y siguen siendo los actores principales del proceso ${ }^{8}$; en segundo lugar, la reducción de su espacio de maniobra para la elaboración de las políticas encomendadas al nivel europeo, por el proceso decisorio donde cada vez son más las materias en las que se aplica el principio mayoritario -y no la unanimidad- - .

Debido a las funciones de los ejecutivos, ha surgido la necesidad de adaptar su estructura a las exigencias de coordinación planteadas por la participación en el contexto europeo y de establecer mecanismos que permitan llegar a una posición compartida de la forma más rápida y eficaz posible ${ }^{10}$.

La evaluación de la eficacia de los diferentes sistemas de coordinación es el objetivo de los estudios politológicos anteriores y actuales, mientras que, desde el punto de vista jurídico, el aspecto a analizar es la estructura de las sedes donde dicha coordinación se lleva a cabo y su eventual desarrollo más o menos paralelo a la evolución de la integración o a la evolución interna del sistema. Queda claro que es beneficioso emplear estos trabajos como instru-

tions and Public Policy. Perspectives on European Decision Making, Kluwer Academic Publisher, La Haya, 1997, p. 159, quien lo describía como la sustitución a largo plazo de prácticas y estructuras preexistentes. Es muy sugestiva la diferenciación entre el concepto de adaptación y los cambios traumáticos realizada por Börzel, T.A., States and Regions in the European Union. Institutional Adaptation in Germany and Spain, Cambridge University Press, Cambridge, 2002, p. 22 y ss.

7 Véase KASSIM, H., «The Europeization of Member State Institutions», en Bulmer, S. y LeQuesne, C. (eds.), The Member States of the European Union, Oxford University Press, Oxford, 2005, p. 285 y ss.

${ }^{8}$ Otra cuestión es la modificación de los equilibrios infra-gubernamentales, es decir, entre los diferentes Ministerios involucrados. La existencia de estos dos fenómenos, diversamente relacionados entre sí, es subrayada por GOETZ, K.H., «European integration and national executives: a cause in search of an effect?», West European Politics, Vol. 23, n. 4, 2000, p. 211 y ss.

9 Sobre esta cuestión, véase LANCHESTER, F., «Introduzione», en ID. (ed.), La barra e il timone. Governo e apparati amministrativi in alcuni ordinamenti costituzionali, Giuffrè, Milán, 2009, p. XVIII.

${ }^{10}$ Decía el Primer Ministro M. Rocard, en una circular del 15 de octubre de 1988, que « l'unité des positions françaises est une condition de l'efficacité de notre action [...] ». Sin embargo, hay también estudios en los que se cuestiona la relación causa-efecto entre una buena organización interna y los resultados en las negociaciones europeas, como SEPOS, A., «The National Coordination of EU Policy: Organisational Efficiency and European Outcomes», Journal of European Integration, Vol. 27, n. 2, 2005, p. 169 y ss. 
mentos supletorios en la investigación, confirmando la faceta acentuadamente multidisciplinar del tema.

\section{METODOLOGÍA: UN ESTUDIO DE DERECHO CONSTITUCIONAL COMPARADO Y EUROPEO}

Las razones para afrontar este tema son múltiples.

En primer lugar, reiteramos que los Gobiernos nacionales han sido siempre y siguen siendo las piezas centrales en la historia de la integración, como protagonistas de toda decisión política y normativa, tanto en la fase ascendente, a la que se va a hacer referencia en el texto, como, cada vez más, en la descendente ${ }^{11}$.

En segundo lugar, el proceso de profundización, especialmente tras la entrada en vigor del Tratado de Lisboa ${ }^{12}$, postula una renovada atención hacia la relación entre la UE y sus Estados miembros. No obstante, debido a la participación creciente de los Parlamentos nacionales, de las Regiones e incluso de los órganos jurisdiccionales, los estudios más recientes se dedican a ellos, más que a los ejecutivos, a pesar de que el eje fundamental no se haya deslizado hacia estos nuevos actores.

Además, la literatura sobre este tema se remonta al principio del $\operatorname{siglo}^{13} \mathrm{y}$ los volúmenes más actualizado no contienen muchos casos de estudio ${ }^{14}$, si se exceptúan los trabajos sobre los miembros recién incorporados de Europa central y del Este, que suelen abarcar un amplio abanico de países ${ }^{15}$. Al con-

${ }^{11}$ Véase la reconstrucción de KASSIM, H., «The Europeanization of Member State Institutions», Ob. Cit., p. 292 y ss.

${ }_{12}$ Los cambios introducidos por este Tratado que tienen reflejos en la posición de los ejecutivos nacionales son numerosos. Entre ellos, se pueden recordar la institución de una Presidencia estable del Consejo Europeo, la creación de la figura del alto representante de asuntos exteriores y política de seguridad y la extensión del procedimiento de co-decisión. Asimismo tienen efectos indirectos sobre las tareas de los ejecutivos el aumento de poderes del Parlamento Europeo, la puesta en marcha de los mecanismos previstos en el protocolo de subsidiariedad y la potencial involucración de los legislativos nacionales en el control.

13 Pienso en particular en Kassim, H., Peters, B.G. y Wright, V. (eds.), The National Co-ordination of EU Policy. The Domestic Level, Ob. Cit..: este trabajo tenía la virtud de incluir a los dos tercios de los Estados miembros en su momento, es decir, Reino Unido, Alemania, Francia, Italia, España, Portugal, Grecia, Bélgica, Austria y Dinamarca.

14 Jordan, A. y Schout, A., The coordination of the European Union. Exploring the capacities of networked governance, Oxford University Press, Oxford, 2006, se concentra sobre Alemania, Países Bajos y Reino Unido; BARONCELli, S. (ed.), Il ruolo del Governo nella formazione e applicazione del diritto dell'Unione europea. Le peculiarità di un sistema costituzionale multilivello, Giappichelli, Turín, 2008, examina sólo a España, Alemania, Austria e Italia.

15 Se pueden recordar, para ofrecer algunos ejemplos, GoETz, K.H., «The New Member States and the EU: Responding to Europe», en Bulmer, S. y Lequesne, C. (eds.), The 
trario, el interés por los miembros fundadores ha ido progresivamente disminuyendo.

En tercer lugar, no se puede evitar constatar la escasez de estudios jurídicos al respecto -aún más clara si se hace referencia a los trabajos de corte comparado-. La doctrina jurídica hasta la fecha se ha centrado en aspectos diferentes de la europeización de la Administración, con especial referencia, por ejemplo, a la convergencia en ámbitos donde ha sido necesario crear Autoridades independientes o adaptar el procedimiento administrativo a los estándares europeos ${ }^{16}$.

A pesar del recelo que parte de la doctrina constitucionalista siente hacia la vertiente puramente «política» del Gobierno y de la Administración, estos aspectos encarnan cuestiones clásicas del Derecho Constitucional, que abarcan tanto aspectos tradicionales, como la composición del Gobierno, la responsabilidad de los Ministros, etc., como aspectos más actuales, relacionados con las variaciones funcionales y estructurales debidas (también) a la integración europea. Por ello, en la perspectiva adoptada aquí, el tema se sitúa en la intersección entre Derecho Constitucional, Derecho Comparado y estudios europeos.

Dos precisiones sobre los términos adoptados en el desarrollo del texto: a) no se hará referencia exclusivamente a los Ministros, pues en algunos casos hay que tener en cuenta también a otros órganos administrativos involu$\operatorname{crados}^{17} \mathrm{y}$ b) la coordinación se va a entender como cualquier actividad regu-

Member States of the European Union, Oxford University Press, Oxford, 2004; DiмiтRoVA, A. y Toshkov, D., «The Dynamics of Domestic Coordination of EU Policy in the New Member States: Impossible to Lock In?», West European Politics, vol. 30, n. 5, 2007; GÄrTnER, L., Hörner, J. y OBHOlzer, L., «National Coordination of EU Policy: A Comparative Study of the Twelve 'New' Member States», Journal of Contemporary European Research, Vol. 7, n. 1, 2011.

${ }^{16}$ Las aportaciones al respecto son muy numerosas. Para citar a algunos autores italianos, véanse, por ejemplo, CASSESE, S. y BignAmI, F. (eds.), Il procedimento amministrativo nel diritto europeo, Giuffrè, Milán, 2004; BATTINI, S. y VeSPERINI, G. (eds.), Lezioni di diritto amministrativo europeo, Giuffrè, Milán, 2006; CHITI, M.P. y GRECO, G. (eds.), Trattato di diritto amministrativo europeo, Giuffrè, Milán, 2007, $2^{\mathrm{a}}$ ed., en varios volúmenes; Della Cananea, G. (ed.), Diritto amministrativo europeo: principi e istituti, Giuffrè, Milán, 2011, $3^{\text {a }}$ ed.; CHITI, M.P., Diritto amministrativo europeo, Giuffrè, Milán, $2011,4^{\mathrm{a}}$ ed. Otra de las cuestiones analizadas desde el principio fue la interacción funcional entre Administración nacional y europea, como lo demuestra la obra de S. CASSESE. Recordamos aquí sólo su ensayo «Divided powers: European administration and national bureaucracies», en ID. (ed.), The European Administration, IIAS y EIPA, Bruselas y Maastricht, 1987.

${ }_{17}$ Sobre la influencia a nivel gubernamental y administrativo, véase FranchINI, C., «L'impatto dell'integrazione comunitaria sulle relazioni al vertice dell'amministrazione. Poteri governativi e poteri amministrativi», Rivista trimestrale di Diritto Pubblico, n. 3, 1991, p. 775 y ss. 
lada por el ordenamiento dirigida a encaminar la política gubernamental hacia una dirección, a través de instrumentos jurídicos y administrativos para coordinar las instancias públicas implicadas y para resolver los conflictos entre ellas. La perspectiva es la interna y no la europea, con lo cual no se tendrán en cuenta las sedes de coordinación en Bruselas.

Una vez aclarada la justificación y definido el marco de la investigación, es ahora necesario explicar las bases metodológicas de la selección de los casos de estudio. Por un lado, esta opción hace posible seguir la evolución que han sufrido los ejecutivos desde los primeros años de las Comunidades Europeas hasta la UE, permitiendo combinar el análisis sincrónico - de la situación de los diferentes países en la actualidad-con el diacrónico. En particular, a lo largo de las décadas los instrumentos de coordinación han sido modificados a la luz de la evolución de la integración, del aumento de los actores involucrados y de las reformas nacionales ${ }^{18}$. Por ello, en las conclusiones se intentará apuntar a los momentos de discontinuidad, con referencia a los factores externos e internos que han influido en los cambios.

Por otro lado, los seis casos constituyen una muestra significativa por algunos factores: a) la organización territorial, pues comprenden a un Estado federal desde siempre (Alemania), a uno que se ha trasformado en Estado federal (Bélgica), a un Estado regional (Italia), y a Estados unitarios con o sin cierta descentralización administrativa (Francia, Países Bajos y Luxemburgo) ${ }^{19}$; b) el peso demográfico y la extensión territorial, que va desde Alemania hasta Luxemburgo; c) las diferentes actitudes hacia la Unión, proactiva y reactiva, y los niveles máximo y mínimo de preocupación por la coordinación (FranciaPaíses Bajos $^{20}$ ). Sin embargo, al mismo tiempo hay un elemento que sustenta esta micro-comparación ${ }^{21}$, es decir, el modelo administrativo común, ya que

${ }_{18}$ Además, hay otro aspecto que mancomuna a estos casos, aunque no esté necesariamente relacionado con la cuestión organizativa: al principio todos los Estados analizados tuvieron que afrontar los mismos retos, el primero de los cuales residía en la falta de personal con una formación específica en asuntos europeos. Sobre el caso francés, BouLOUIS, J., «La incidencia del Derecho Comunitario en la Organización Administrativa Francesa», Revista Andaluza de Administración Pública, n. 9, 1992, y sobre la importancia de los funcionarios y empleados públicos, véase en particular CARDONA, F., «Civil services for the European Administrative Space», Public Management Forum, n. 2, 1999.

${ }^{19} \mathrm{CH}$. DebBasch, «L'influence du processus d'integration communautaire sur les administrations nationales», Rivista Trimestrale di Diritto Pubblico, n. 3, 1991, p. 743 y ss., adopta el elemento territorial como criterio clasificatorio. Asimismo ORDóÑEz Solís, D., «Administraciones nacionales e integración europea», Noticias de la Unión Europea, n. 136, 1996, p. 19 y ss., lo considera un factor fundamental.

${ }^{20}$ La opinión es de Kassim, H., Peters, B.G. y Wright V. (eds.), The National Coordination of EU Policy. The Domestic Level, Ob. Cit., p. 3.

${ }^{21}$ Véase, en castellano, SCARCiglia, R., «Macrocomparación/Microcomparación», en Pegoraro, L. (ed.), Glosario de Derecho Público Comparado, Porrúa, México, 2012, p. 280. 
todos los Estados analizados forman parte de la tradición continental, no habiendo sistemas anglosajones o escandinavos, por ejemplo.

En la aplicación del método comparado, la investigación tendrá en cuenta dos de los tres formantes ${ }^{22}$ : el legal (relativo a las normas sobre la organización de las sedes de coordinación) y el doctrinal, mientras que no tiene especial trascendencia el jurisprudencial ${ }^{23}$.

Coherentemente con la finalidad primigenia de la comparación jurídica, de tipo teórico cognoscitivo ${ }^{24}$, clasificaremos los sistemas en los $\langle\text { modelos }\rangle^{25}$ derivados de la aplicación de los siguientes «elementos determinantes»» ${ }^{26}$ : a) la existencia o inexistencia de un Ministro para los Asuntos Europeos; b) la existencia o inexistencia de un órgano de coordinación interministerial específico y b1) su colocación en la Presidencia del Gobierno o en el Ministerio de Asuntos Exteriores.

A la luz de las diferencias en el papel de los miembros del ejecutivo ${ }^{27}$, hasta la fecha la literatura ha hablado básicamente de dos patrones: los siste-

${ }^{22}$ Este término se debe a la obra de Rodolfo SACCO (véase Introduzione al diritto comparato, Utet, Turín, 1992, p. 43 y ss.) y es utilizado en muchos de los estudios sobre el método en el Derecho Público Comparado, como BognetTI, G., Introduzione al diritto costituzionale comparato, Giappichelli, Turín, 1994, p. 82 y ss.; Pegoraro, L. y Rinella, A., Diritto costituzionale comparato. Aspetti metodologici, Padua, Cedam, 2013, p. 38 y ss.; SCARCiGLIA, R., Introducción al Derecho Constitucional Comparado, Dykinson, Madrid, 2011, p. 116 y ss.

${ }^{23}$ Una precisión: el papel de la jurisprudencia nacional y del Tribunal de Justicia ha sido y es esencial para la evolución de la UE y, en algunos casos, para el establecimiento de los equilibrios interinstitucionales. Sin embargo, no hay material relevante con referencia específicamente a los instrumentos de coordinación.

${ }^{24}$ Sobre las finalidades del Derecho Comparado, se pueden recordar LOMBARDI, G., Premesse al corso di diritto pubblico comparato, Giuffrè, Milán, 1986, p. 11 y ss.; De VerGOTTINI, G., Diritto costituzionale comparato, Cedam, Padua, 2011, $8^{\text {a }}$ ed., p. 16 y ss. y PeGORARo, L. y Rinella, A., Diritto costituzionale comparato. Aspetti metodologici, cit., p. 72 y ss.

${ }_{25}$ Véase al respecto Tusseau, G., «Modelos», en Pegoraro, L. (ed.), Glosario de Derecho Público Comparado, Ob. Cit., p. 288: «La comparación jurídica dirige la atención del jurista sobre una multiplicidad de Estados, instituciones, normas, etc.; con su confrontación, él llega a constataciones que varían por la oposición a la identidad, pasando por la diferencia, la semejanza o la equivalencia. La ambición de la empresa del comparatista no consiste únicamente en tomar nota de la diversidad o la proximidad de los elementos comparados. La contribución original, de hecho, se encuentra más bien en proceder a una mise en ordre intelectual de los datos examinados, que representa el objetivo de la construcción de modelos».

${ }^{26}$ Este método se debe a L.J. COnSTANTINESCO, quien lo aplicó al estudio de las familias jurídicas y de las formas de Estado, aunque puede ser adoptado también en el ámbito de estudios sobre objetos más reducidos. Véase CONSTANTINESCO, L.J., Introduzione al diritto comparato, Giappichelli, Turín, 1996; ID., Il metodo comparativo, edición de ProCIDA Mirabelli Di Lauro, A., Giappichelli, Turín, 2000.

27 Sobre la ausencia de un modelo único, además de los otros escritos citados infra, véase Mittag, J. y Wessels, W., «The 'One' and the 'Fifteen'? The Member States bet- 
mas dominados por el Ministro de Asuntos Exteriores y los sistemas dominados por el Primer Ministro ${ }^{28}$. Empero estas clasificaciones hacen referencia precipuamente a factores políticos, como los equilibrios entre Ministerios, el protagonismo de los Ministros y del Presidente del Gobierno en la praxis, etc.

En esta investigación, al contrario, a través de los «elementos determinantes» mencionados con anterioridad, que hacen referencia al formante legal, se llegará a incluir a los países analizados en cuatro modelos. Su elaboración se va a realizar de forma progresiva, desde los casos que reflejan en menor medida los elementos considerados síntomas de desarrollo del sistema, es decir, existencia de un Ministro para los Asuntos Europeos y presencia de una sede de coordinación especializada. En este segundo ámbito, siendo el alejamiento de la sede de coordinación del Ministerio de Asuntos Exteriores una supuesta tendencia, la opción menos desarrollada será su presencia dentro de dicho Ministerio.

El primer modelo se denominará así «opción cero» y estará caracterizado por la falta de un Ministro o de una sede (Alemania, § 4.1); en el segundo tampoco hay un Ministro para los Asuntos Europeos, pero sí una sede de coordinación en el Ministerio de Asuntos Exteriores (Bélgica, Países Bajos y Luxemburgo, § 4.2); en el tercero dicha sede se sitúa en la Presidencia del Gobierno (Francia, § 4.3); finalmente, en el cuarto se cumplen las dos condiciones, es decir, la existencia de un Ministro específico y de una sede en la Presidencia (Italia, § 4.4).

En cada párrafo se podrá mencionar a las unidades o divisiones dentro de los Ministerios, aunque no se va a profundizar en ellas a no ser que resulten determinantes para la coordinación. Sólo en el análisis del caso belga resultará necesario involucrar el aspecto territorial en el examen de la coordinación interministerial porque es uno de los rasgos esenciales del sistema, mientras que por lo demás se van a omitir las referencias a los actores nuevos (todavía secundarios) del escenario europeo: Parlamentos y Regiones.

Antes de pasar a una breve reseña de los sistemas de coordinación que abarque un mayor número de ejemplos $(\S 3)$, es necesaria una última precisión metodológica con referencia a las categorías propuestas. Se va a realizar una clasificación politética, basada en una pluralidad de parámetros, que no

ween procedural adaptation and structural revolution», en WeSSELS, W., MAURER, A. y Mittag, J. (eds.), Fifteen into one? The European Union and its member states, Manchester University Press, Manchester-Nueva York, 2003, p. 413 y ss.

${ }^{28}$ LAfFAn, B., «Managing Europe from Home. Impact of the EU on Executive Government: A Comparative Analysis», OEUE Paper, 2003, en www.oeue.net, habla de dos modelos preponderantes, con referencia al papel del Primer Ministro o del Ministro de Asuntos Exteriores; CIAVARINI AzZI, G., «L'application du Droit communautaire dans les États membres: une vue d'ensemble», Revue Française d'administration publique, n. 34, 1985, elaboraba tres modelos. 
asume ninguno por sí solo como elemento decisivo (como pasa en las monotéticas) y es, por lo tanto, más flexible ${ }^{29}$. Adoptando la lógica difusa (fuzzy sets theory, en su denominación originaria elaborada por L.A. Zadeh), se propondrán así clases difuminadas, en las cuales los objetos adscritos a cada una presentan también alguna característica divergente, además de compartir aquellas que resulten determinantes para la clasificación. A diferencia de las ciencias exactas, las clases en el Derecho Comparado son menos netas, tanto porque presentan propiedades multiformes e interpretables, como porque están sujetas a caducidad por la misma evolución de los ordenamientos.

\section{SOLUCIONES COMPARADAS: UNA OJEADA RÁPIDA}

La dimensión europea de los Gobiernos nacionales ha sido en parte obviada en los estudios más recientes, pues la doctrina está dedicando más atención a aquellos sujetos que han tardado en conseguir «subir al escenario» europeo, los legislativos y los entes descentralizados ${ }^{30}$.

Sin embargo, tener en cuenta los estudios anteriores relativos a los ejecutivos resulta útil porque éstos han individualizado algunas tendencias generalizadas. Nos referimos, por ejemplo, a la creación de estructuras gubernamentales especializadas en cuestiones europeas ${ }^{31} \mathrm{y}$ a la distinción paulatina entre asuntos exteriores y asuntos europeos ${ }^{32}$-aunque la PESC sigue dentro del primer campo-. Se puede añadir la persistente importancia de las Administraciones centrales, que reciben en primera instancia todas las informaciones de la UE y son las titulares de la casi totalidad de los instrumentos participativos a nivel europeo. Asimismo, el alma económica de la Unión -y la reciente crisis- parece haber afectado de manera bastante pareja a los Estados miembros en el sentido de revalorizar las funciones de los Ministerios de

${ }^{29}$ Sobre la aplicación de esta teoría en el Derecho Comparado, véase la reciente reconstrucción de BALDIN, S., «Riflessioni sull'uso consapevole della logica fuzzy nelle classificazioni fra epistemologia del diritto comparato e interdisciplinarietà», Revista General de Derecho Público Comparado, n. 10, 2012.

30 Véanse Kassim, H., Peters, B.G. y Wright, V. (eds.), The National Co-ordination of EU Policy. The Domestic Level, Ob. Cit.; Wessels W., Maurer, A. y MitTaG, J. (eds.), Fifteen into one? The European Union and its Member States, Manchester University Press, Manchester, 2003; JoRDAN, A. y SchOUT, A. (eds.), The Coordination of the European Union. Exploring the Capacities of a Networked Governance, Oxford University Press, Oxford, 2006.

${ }^{31}$ Sobre este efecto, véase DeBbasch, $\mathrm{CH}$. «L'influence du processus d'integration communautaire sur les administrations nationales», Ob. Cit., p. 742.

32 Estas dos tendencias son evidenciadas por Closa Montero, C. y Molina ÁlvaReZ DE Cienfuegos, I., «La elaboración y coordinación de la política europea: apuntes para negociar mejor la maraña post-Lisboa», en www.realinstitutoelcano.org, ARI 121/2010, p. 1 . 
Economía y más, cuando están separados, los de Hacienda ${ }^{33}$. Finalmente, una tendencia bastante clara a lo largo de los años resulta ser la potenciación de las funciones directivas y representativas del Primer Ministro, tal y como el papel coordinador de la Presidencia en ii $^{34}$.

A pesar de estas tendencias compartidas, sigue habiendo sistemas diferenciados desde el punto de vista estructural ${ }^{35}$.

Sólo para mencionar algunos casos, en Suecia y en Finlandia se han instituido Ministros ad hoc para los asuntos europeos ${ }^{36}$, y en muchos casos se ha encargado de seguir estas cuestiones a un secretario de Estado, un viceministro o un Junior Minister ${ }^{37}$.

A nivel administrativo, como se ha anticipado, el órgano de coordinación puede colocarse dentro de la Presidencia del Gobierno o del Ministerio de Asuntos Exteriores (o, eventualmente, de otro Ministerio). Por ejemplo, la Secretaría para Asuntos Europeos y globales del Reino Unido está conectada a la Oficina del Primer Ministro ${ }^{38}$, mientras que la Comisión Interministerial para los Asuntos Europeos portuguesa está vinculada al Ministerio de Asuntos Exteriores ${ }^{39}$ y la Secretaría de Estado para la Unión

${ }^{33}$ Goetz, K.H. y Meyer-Sahling, J.H., «The Europeanisation of national political systems: Parliaments and executives», Ob. Cit., p. 14.

${ }^{34}$ En este sentido, véase Mittag, J. y Wessels, W., «The 'One' and the 'Fifteen'? The Member States between procedural adaptation and structural revolution», Ob. Cit., p. 424 y ss.

35 Olsen, J.P., Europe in Search of Political Order, Oxford University Press, Oxford, 2007 , p. 239, lo pone de manifiesto de forma muy clara: «While there are competing interpretations, the main findings (although with many nuances) are, first, that ,Europe matters' and second, that there is domestic persistence and enduring diversity [...]. There has been no general trend towards isomorphism and no significant convergence towards a common institutional model homogenizing the domestic structures of the European states [...] established domestic patterns have been resilient but also flexible enough to cope with changes at the European level, and no new and unified model of dealing with Union matters has emerged».

${ }^{36}$ Para conseguir mayores informaciones, se pueden consultar las páginas web de los dos Gobiernos: www.sweden.gov.se y www.government.fi.

37 Closa Montero, C. y Molina Álvarez de Cienfuegos, I., «La elaboración y coordinación de la política europea: apuntes para negociar mejor la maraña post-Lisboa», Ob. Cit., p. 4.

${ }^{38}$ Véase la página web del European and Global Issues Secretariat, www.cabinetoffice.gov.uk. Entre las funciones encomendadas a esta Secretaría, se encuentra el apoyo al Gobierno en la gestión y preparación de las reuniones del Consejo Europeo, de las negociaciones y de las relaciones con los demás Estados miembros (y candidatos), además de la coordinación entre Administraciones en el proceso decisorio y de ejecución de los actos normativos europeos. Sobre la evolución de la coordinación en el Reino Unido, véase Kassim, H., «United Kingdom», en Kassim, H., Peters, B.G. y Wright, V. (eds.), The National Co-ordination of EU Policy. The Domestic Level, Op. Cit., p. 22 y ss.

39 Véase la página web de la Dirección General de Asuntos Europeos, www.portugal. gov.pt. Acerca de los instrumentos de coordinación, véase MAGONE, J., «Portugal», en 
Europea española se encuentra en el Ministerio de Asuntos Exteriores y de Cooperación ${ }^{40}$.

\section{LA COORDINACIÓN GUBERNAMENTAL}

\subsection{Modelo 1: la opción cero, es decir, la coordinación sin Ministro o sede especifica}

Tal y como se explicó con anterioridad, en el primer modelo de referencia no hay ni un Ministro para los Asuntos Europeos ni una sede de coordinación como un Secretariado de corte francés o un Comité a la inglesa.

Y justamente esta «ausencia» es el punto de partida de todo análisis acerca del sistema de coordinación alemán. La adaptación del ejecutivo al proceso de integración no conllevó grandes cambios estructurales, y al principio los dos Ministerios que se peleaban las tareas en ámbito europeo fueron el de Asuntos Exteriores y el de Economía. El compromiso alcanzado en 1958 fue el siguiente: el primero sería competente para las cuestiones relativas a los tratados y a sus reformas, incluida la ampliación; el segundo se ocuparía de todas las materias otorgadas a las entonces CEE. Por ello, dentro de los dos aparatos se crearon divisiones para los asuntos europeos ${ }^{41}$.

El Ministerio de Agricultura también estableció contactos autónomos con los sujetos involucrados desde el principio, y esta tendencia se expandió a los demás Ministerios después de la entrada en vigor del Tratado de Maastri-

Kassim, H., Peters, B.G. y Wright, V. (eds.), The National Co-ordination of EU Policy. The Domestic Level, Ob. Cit., p. 141 y ss.

${ }^{40}$ La actual organización de la Secretaría se debe al Real decreto 342/2012, de 10 de febrero, «por el que se desarrolla la estructura orgánica básica del Ministerio de Asuntos Exteriores y de Cooperación» (en www.maec.es). Conforme al art. 6.1, se trata del «órgano superior directamente responsable, bajo la dirección del titular del Departamento, de la formulación y ejecución de la política de España en el ámbito de la Unión Europea» que además «asiste al titular del Ministerio en la formulación y ejecución de la política exterior de España en el área geográfica correspondiente a los países de la Unión Europea, países candidatos, países del Espacio Económico Europeo y otros países europeos». A su vez, el Secretario de Estado para la Unión Europea preside la Comisión Interministerial para Asuntos relacionados con la Unión Europea (CIAUE), que representa la sede de coordinación entre los Ministerios para definir las directrices de la política europea. Sobre la evolución del sistema, se remite a Molina Álvarez de Cienfuegos, I., «Spain», en Kassim, H., Peters, B.G. y Wright, V.(eds.), The National Co-ordination of EU Policy. The Domestic Level, Ob. Cit., p. 114 y ss. y Closa Montero, C. y Heywood, P.M., Spain and the European Union, Basingstoke, Palgrave Macmillan, 2004, p. 60 y ss.

${ }^{41}$ Sobre la primera fase, véase Derlien, H.U., «Germany», en Kassim, H., Peters, B.G. y Wright, V. (eds.), The National Co-ordination of EU Policy. The Domestic Level, Ob. Cit., p. 58 y ss. 
$\mathrm{cht}^{42}$, aunque todavía se podía ver una clara prevalencia del de Economía. Posteriormente, éste vio disminuir sus tareas a favor del de Hacienda: fue un giro importante cuando, en 1998, muchas competencias fueron transferidas del primero al segundo ${ }^{43}$, aunque después de las elecciones de 2005 la situación volvió sustancialmente al principio ${ }^{44}$.

El sistema se basa en una responsabilidad compartida, que depende del papel del Ministerio competente que se encarga de la coordinación horizontal ${ }^{45}$. Éste tiene que emprender las negociaciones internas para alcanzar una posición común, informar a los demás Ministerios afectados, mantener al día al Parlamento federal y preparar los documentos para el Gobierno federal de cara a la negociación en Bruselas. Finalmente, el Consejo de Ministros representa la sede decisoria más elevada.

Se han instituido mecanismos para facilitar el proceso de coordinación a nivel administrativo ${ }^{46}$ : tras la abolición del Comité para los Asuntos Europeos en la Presidencia ${ }^{47}$, el órgano de referencia es el Comité de los Secretarios de Estado para los Asuntos Europeos (Staatssekretärsausschuss

${ }^{42}$ Véase al respecto MAURER, A., "Germany: fragmented structures in a complex system», en Wessels, W., Maurer, A. y Mittag, J. (eds.), Fifteen into one? The European Union and its Member States, Ob., Cit., p. 125.

${ }^{43}$ El Ministerio asumió la vicepresidencia del Comité de los Secretarios de Estado para los Asuntos Europeos, la coordinación de las reuniones del COREPER I y II y la gestión de los fondos estructurales y de cohesión. Véase en particular BulMER, S. y BuRCH, M., "The Europeanisation of Central Government: the UK and Germany in historical institutionalist perspective», en SCHNEIDER, G. y AsPINwALL, M. (eds.), The Rules of Integration: Institutionalist Approaches to the study of Europe, Manchester University Press, Manchester, 2001, p. 73 y ss.

${ }^{44}$ Goetz, K.H. y MeYer-SAhling, J.H., «The Europeanisation of national political systems: Parliaments and executives», Ob. Cit., p. 14.

${ }^{45}$ Cada Ministerio es un actor independiente, dentro de un contexto caracterizado por la preeminencia del Canciller y de su dirección política. En este sentido, MAURER, A., «Germany: fragmented structures in a complex system», Ob. Cit., p. 119. Véanse también las observaciones contenidas en «Germany: a reactive and passive coordinator?», en JoRDAN, A. y SCHOUT, A., The coordination of the European Union. Exploring the capacities of networked governance, Ob. Cit., p. 149 y ss. - aunque el estudio de caso se refiere a la política de medio ambiente -. Sobre la ausencia de un Ministerio específico, ZEI, A., «L'organizzazione del Governo in Germania», en LANCHESTER, F. (ed.), La barra e il timone. Governo e apparati amministrativi in alcuni ordinamenti costituzionali, Ob. Cit., p. 223 y ss.

46 Derlien, H.U., «Germany», Ob. Cit., p. 55 y ss. hablaba de dos vías: la diplomática, que parte del Ministerio de Asuntos Exteriores y llega hasta el Consejo de Ministros, y la especializada, que se basa en la burocracia alemana y alcanza por un lado a los Länder y por el otro lado a la Comisión.

${ }^{47}$ Dicho órgano fue instituido en 1973 y fue abolido por el Canciller Schröder, puesto que antes del comienzo de su primer mandato (en 1998) sólo había celebrado dos reuniones. Véase al respecto SAsse, C., Regierungen, Parlamente, Ministerrat: Entschei- 
für Europafragen), cuyo Presidente es el Secretario de Estado para Europa del Ministerio de Asuntos Exteriores y el Vice-presidente es el Ministro de Economía. Dicho órgano fue creado en 1963 como sede para debatir las cuestiones europeas cuando los Ministerios afectados no alcanzasen un acuerdo.

El modelo variable de coordinación interministerial y la citada falta de un Ministerio específico han conllevado una mayor sectorialización y han propiciado aún más el desarrollo de sub-divisiones internas a los Ministerios. Los de Agricultura, Hacienda y Asuntos Exteriores crearon Direcciones generales para los Asuntos Europeos ya en los años 60 y los siguieron otros; las reuniones entre los vértices de dichas Direcciones también se celebran cada mes aproximadamente en la sede del Ministerio de Asuntos Exteriores o en el de Economía, para afrontar los eventuales casos de alerta temprana, resolver los contrastes entre las Administraciones involucradas (si es posible) y monitorizar la ejecución de las normas europeas ${ }^{48}$. También se dan encuentros de los Jefes de los Asuntos Europeos - que suelen ser las cabezas de la división de coordinación de cada Dirección-con funciones de apoyo a los demás órganos mencionados, sobre todo al Comité de los Secretarios de Estado para los Asuntos Europeos.

\subsection{Modelo 2: la coordinación sin Ministro, pero con una sede en el Ministerio de Asuntos Exteriores}

Dentro de esta segunda categoría, más «tradicional» porque reconoce un papel determinante al Ministerio de Asuntos Exteriores, se pueden incluir a Bélgica, Países Bajos y Luxemburgo.

Como se aclaró, en el caso belga resulta esencial la transformación de la forma de Estado.

En las primeras décadas, además de la adaptación interna de cada vez más Ministerios, mediante la creación de divisiones y direcciones ${ }^{49}$, el sistema de coordinación evolucionó otorgando cada vez más protagonismo a la Dirección general para los asuntos políticos (situada dentro del Ministerio de Asuntos Exteriores). Para favorecer la cooperación, este mecanismo, denominado

dungsprozesse in der Europäischen Gemeinschaft, Europa Union Verlag, Bonn, 1975, p. 28 y ss.

${ }^{48}$ En particular, el grupo de coordinación europea que forma parte de la Dirección general del Ministerio de Asuntos Exteriores desempeña tareas de monitorización sobre las iniciativas europeas, su relación con las políticas alemanas y la formación de las posiciones nacionales.

49 Kerremans, B., Beyers, J., «Belgium: The Dilemma between Cohesion and Autonomy», en Hanf, K., Soetendorp, B. (eds.), Adapting to European integration: small States and the European Union, Longman, Londres, 1998, p. 17 y ss. 
«Coordinación europea» entre 1974 y 1985 y luego «P.11», a su vez se coordinaba con el Comité económico interministerial situado en el de Economía.

El punto de partida para el análisis del sistema de coordinación belga actual -es decir, en la fase federal posterior a 1993- reside en la complejidad de la organización territorial (con la presencia de tres Regiones y tres Comunidades lingüísticas) ${ }^{50}$. La coordinación pasó entonces de ser básicamente un problema del Gobierno nacional a ser una cuestión interterritorial, dado que desde el punto de vista jurídico, los ejecutivos regionales empezaron a tener el mismo rango que el estatal y se inició a emplear el principio de unanimidad ${ }^{51}$. Estas reformas «han permitido desarrollar unos sistemas de participación de las Regiones tanto en la fase ascendente como en la descendente que en muchos casos posterga la intervención del Gobierno y de la Administración central $\rangle^{52}$.

Así se potenció el alcance del principio in foro interno in foro externo, según el cual los entes descentralizados pueden desempeñar funciones de política exterior si disponen de la competencia a nivel interno sobre la materia afectada. Se fortalecieron los mecanismos dirigidos a garantizar el flujo de informaciones hacia los entes y a propiciar la cooperación, especialmente en las competencias compartidas ${ }^{53}$.

El posterior acuerdo adoptado por las Comunidades y Regiones en 1994 acerca de la coordinación en los asuntos europeos determinó también qué territorio representaría al Estado en los Consejos. El acuerdo fue actualizado en 2003, tras la reforma de 2001 que aumentó las competencias regionales: se estableció un sistema basado en seis grupos de materias, con una rotación semestral entre Comunidades y Regiones ${ }^{54}$.

En ausencia de un Ministerio específicamente dedicado a los asuntos europeos, la sede más importante de coordinación interna es la Dirección General para la Coordinación y los Asuntos Europeos (Direction générale Coordination et Affaires européennes - DGE $)^{55}$, situada en el Ministerio de

${ }^{50}$ Beyers, J., Kerremans, B. y Bursens, P., «Belgium, the Netherlands, and Luxembourg: Diversity Among the Benelux Countries», Ob. Cit., p. 63.

51 Véase Kerremans, B., «Belgium», en Kassim, H., Peters, B.G. y Wright, V. (eds.), The National Co-ordination of EU Policy. The Domestic Level, Ob. Cit., p. 186 y ss.

${ }^{52}$ OrdóÑEZ Solís, D. «Administraciones nacionales e integración europea», cit., p. 39. Como lo afirman Kerremans, B. y Beyers, J., «Belgium: The Dilemma between Cohesion and Autonomy», Ob. Cit., «Belgium can only take a position in the Council if both the federal government and each subnational level have approved that position».

53 Véanse Drieskens, E., Van Hecke, S. y Bursens, P., The 2010 Belgian Presidency: Driving in the EU's Back Seat, Swedish Institute for European Policy Studies, Estocolmo, 2010, pp. 28-29.

${ }^{54}$ Se trataba del Accord de coopération entre l'Etat fédéral, les Communautés et les Régions, relatif à la représentation du Royaume de Belgique au sein du Conseil de Ministres de l'Union européenne del 17 de noviembre de 1994, reformado el 13 de febrero de 2003.

55 Sobre su organización interna se puede consultar la página web de la Dirección: http://diplomatie.belgium.be/fr/sur_lorganisation/organigramme_et_structure/e/. 
Asuntos Exteriores. Dentro de dicha institución se prepara y define la posición belga, a través de reuniones periódicas con representantes de los ejecutivos involucrados. La naturaleza de esta Dirección se ha ido modificando a lo largo de los años, justamente porque la participación de sujetos provenientes de los otros niveles de gobierno, cuyo voto tiene el mismo valor que el federal, la ha trasformado en una «agencia inter-federal con una lógica federal dual definida en la Constitución $»^{56}$.

Si no se llega a un acuerdo, interviene la Conferencia Interministerial para la Política Exterior (Conférence Interministérielle «Politique étrangère»-CIPE), que está compuesta por los Ministros competentes del Estado y de las Regiones y Comunidades. Eventualmente se puede llegar posteriormente al Comité de Concertación (Comité de concertation), que se sitúa en la Presidencia del Gobierno y está formado igualmente por Ministros de los diferentes niveles territoriales. Se trata del órgano de coordinación general y por ello se podría decir que las Conferencias interministeriales son una suerte de emanación de este Comités7.

Pasando al segundo ordenamiento que encaja en esta clase, es decir, el sistema holandés, éste presenta dos características fundamentales: el protagonismo del Ministerio de Asuntos Exteriores y el mantenimiento de la fragmentación en la burocracia y de cierta autonomía para los Ministerios ${ }^{58}$.

En los primeros años, hasta 1972, el papel coordinador le pertenecía al Ministerio de Economía, pero a partir de esta fecha pasó al Ministerio de Asuntos Exteriores, dentro del cual se encuentra la Dirección general para la cooperación europea. Las iniciativas de la Comisión se comunican a la representación permanente, que a su vez las transmite a este Ministerio: posteriormente, se identifica al Ministerio competente, que se encarga tanto de la fase ascendente como de la descendente y además prepara el primer borrador de la posición nacional a defender ante las Instituciones europeas.

Cada Ministerio dispone de un departamento y/o división especializada y hay reuniones de los sub-directores generales de cada uno (de los afectados), bajo la dirección del Secretario de Estado de Asuntos Exteriores ${ }^{59}$.

${ }^{56}$ Drieskens, E., Van Hecke, S. y Bursens, P., The 2010 Belgian Presidency: Driving in the EU's Back Seat, Ob. Cit., p. 32.

${ }^{57}$ Una imagen gráfica de estos tres niveles, Dirección general - Conferencia interministerial - Comité de concertación, se encuentra en BEYERS, J. y Bursens, P., «The European Rescue of the Federal State. How Europeanization Shapes the Belgian State», West European Politics, Vol. 29, n. 5, 2006, p. 1057 y ss.

58 Para una exposición extensa de estos caracteres tradicionales, véase HARMSEN, R., «The Europeanization of National Administrations: A Comparative Study of France and The Netherlands», Governance, Vol. 12, n. 1, 1999, p. 81 y ss.

${ }^{59} \mathrm{Si}$ bien es verdad que la responsabilidad de la coordinación radica en este Ministerio, su papel efectivo es más bien débil, llegando a ser definido como un weak integrator. Véase, aparte de la literatura mencionada infra, «The Netherlands: from event to issue 
Las decisiones de los órganos de coordinación pueden llegar al Consejo de Ministros $^{60}$, pues el sistema funciona como una cadena de apelaciones progresivas, en la cual los Ministerios intentan sostener su punto de vista en cada etapa ${ }^{61}$.

Dentro de esta clase, se puede incluir también a Luxemburgo. Su sistema de coordinación es el más sencillo, debido a los caracteres peculiares de este Estado miembro. El Ministerio que recibe las iniciativas de la Comisión -a través de la representación permanente- es el de Asuntos Exteriores, quien a su vez las distribuye entre los diferentes Ministerios según la materia afectada. En caso de cuestiones importantes o que afecten a diversos departamentos, se demandan a un Comité de la Unión Europea, presidido por el Ministro de Asuntos Exteriores, donde participan los Ministros afectados, el Primer Ministro y la REPER ${ }^{6}$.

\subsection{Modelo 3: la coordinación sin Ministro, pero con una sede en la Presidencia del Gobierno}

El sistema francés es un ejemplo claro de coordinación especializada. La Secretaría General de Cooperación Interministerial (SGCI), creada en 1948 para resolver los conflictos entre el Ministerio de Asuntos Exteriores y el de Hacienda en la gestión de las ayudas del Plan Marshall, se ha revelado esencial para el desempeño de las tareas europeas del Gobierno. Ésta coordinaba las diferentes Administraciones y dependía tradicionalmente de la Presidencia (aunque desde 1981 hasta 1984 se adscribió al Viceministro para los Asuntos Europeos) $)^{63}$.

coordination», en Jordan, A. y Schout, A., The coordination of the European Union. Exploring the capacities of networked governance, Ob. Cit., p. 166 y ss.

${ }^{60}$ Sobre su papel, véase SoETENDORP, B. y Hanf, K., «The Netherlands: Growing Doubts of a Loyal member», en HAnF, K. y SoETEndorP, B. (eds.), Adapting to European integration: small States and the European Union, Ob. Cit., p. 40.

${ }_{61}$ Sobre los diferentes niveles y su relación, véase Beyers, J., Kerremans, B. y BurSENS, P., «Belgium, the Netherlands, and Luxembourg: Diversity Among the Benelux Countries», en E.E. ZefF, E.B. Pirro (eds.), The European Union and the Member States. Cooperation, Coordination, and Compromise, Rienner, Londres, 2001, p. 60 y ss.

${ }^{62} \mathrm{Su}$ papel de coordinación es mínimo, según Beyers, J., Kerremans, B. y Bursens, P., «Belgium, the Netherlands, and Luxembourg: Diversity Among the Benelux Countries», Ob. Cit., p. 72, que enfatizan los contactos informales.

${ }^{63}$ Para un análisis histórico de las posiciones relacionadas con las distintas políticas, véase HAYWARD, J. y Wright, V., Governing from the centre. Core executive coordination in France, Oxford University Press, Oxford, 2002, p. 133 y ss. En general, sobre la actitud de los Gobiernos franceses, véase J.S. AMBLER, M.S. REICHERT, «France: Europeanism, Nationalism, and the Planned Economy», en ZEFF, EE. y PIRro, E.B. (eds.), The European Union and the Member States. Cooperation, Coordination, and Compromise, Ob. Cit., p. 29 y ss. 
En 2005 se aprobó la última regulación de este órgano ${ }^{64}$ y desde entonces el sistema se basa en la Secretaría General para los Asuntos Europeos (SGAE), ubicada en la Presidencia del Gobierno ${ }^{65}$. Toda la fase ascendente es dominada por ella, que recibe las iniciativas de la Comisión, las transmite a los Ministerios afectados y organiza los encuentros entre ellos, además de tener el monopolio en las relaciones con la representación permanente francesa ${ }^{66}$.

Bajo la autoridad del Primer Ministro, ésta instruye y prepara las posiciones a defender en el Consejo de la Unión Europea (pero se ocupa asimismo de la fase descendente junto con la Secretaría general del Gobierno) y transmite la cuestión a la Presidencia si no se alcanza un acuerdo.

Se ha instaurado además el Comité interministerial sobre Europa, que reúne los principales Ministros y Secretarios de Estado sobre la base de un orden del día decidido por el Primer Ministro; la labor de apoyo le corresponde a la SGAE. Los Ministros que forman parte de dicho Comité son el de Asuntos Exteriores, el de Economía y Hacienda, el Vice-Ministro delegado por el Ministro de Asuntos Exteriores para las cuestiones europeas y los demás interesados según la materia.

\subsection{Modelo 4: la coordinación con Ministro y con una sede en la Presidencia del Gobierno}

En el caso italiano también los instrumentos institucionales y administrativos dirigidos a la coordinación de las políticas europeas se han modificado

${ }^{64}$ Decreto 2005-1283, «relatif au comité interministériel sur l'Europe et au secrétariat général des affaires européennes». Sobre la SGCI, véanse Menon, A., «France», en Kassim, H., Peters, B.G. y Wright, V. (eds.), The National Co-ordination of EU Policy. The Domestic Level, Ob. Cit., p. 83 y ss. y Szukala, A., «France: the European transformation of the French model», en Wessels, W., Maurer, A. y Mittag, J. (eds.), Fifteen into one? The European Union and its Member States, Ob. Cit., p. 228 y ss.

${ }_{65}$ Véase la página web del Secrétariat général des affaires européennes, www.sgae. gouv.fr. En la descripción de sus funciones, se hace referencia a todas las áreas incluidas en el Tratado sobre el Funcionamiento de la Unión Europea (menos la PESC) y a las relacines con la REPER francesa.

${ }^{66}$ En el art. 2 del decreto 2005-1283 se afirma, por ejemplo, que la secretaría general, bajo la autoridad del primer ministro, « instruit et prépare les positions qui seront exprimées par la France au sein des institutions de l'Union européenne [...]. Il assure la coordination interministérielle nécessaire à cet effet. Il transmet les instructions du Gouvernement aux agents chargés de l'expression des positions françaises auprès de ces institutions »; además " veille à la mise en oeuvre, par l'ensemble des départements ministériels, des engagements souscrits par le Gouvernement dans le cadre des institutions européennes »; etc. Ésta se encarga asimismo de monitorizar la trasposición de las directivas y la participación francesa en las Instituciones europeas. 
a lo largo de las décadas ${ }^{67}$. La doctrina ha hablado de dos etapas: a) la de la primacía del Gobierno central, y especialmente del Ministro de Asuntos Exteriores; b) la de la fragmentación, con un creciente papel de diferentes Administraciones ${ }^{68}$. Hasta los años 80 no existían formas de participación de las Regiones en los asuntos comunitarios (era la época de la «ceguera regional» europea, y el Estado por su parte no introdujo enmiendas al respecto) ni instrumentos para que el Parlamento pudiera ser involucrado en la definición de la política europea o controlar la actuación del Gobierno.

En esta primera fase no se reformó la estructura gubernamental y se encomendó al Ministro de Asuntos Exteriores la gestión de todo lo relacionado con las Comunidades Europeas ${ }^{69}$. Su papel se vio reducido en la segunda fase por dos razones básicas: a) la potenciación de los diferentes Ministerios en la gestión de las cuestiones europeas en sedes cooperativas; b) la creación, en 1987, del Departamento para la Coordinación de las Políticas Comunitarias ${ }^{70}$ dirigido por un Ministro sin cartera que tenía como funciones la coordinación y el seguimiento de la implementación de las directivas.

Entre la sedes específicas de coordinación, la primera fue el Comité Interministerial para la Programación Económica (CIPE), creado en 1967 en el seno de la Presidencia del Gobierno ${ }^{71}$; tenía funciones relativas a la política

${ }^{67}$ Se nos permita remitir a nuestro «Coordinación de las políticas europeas y transformaciones estructurales de los Ejecutivos nacionales (el caso italiano en perspectiva comparada)», Revista General de Derecho Público Comparado, n. 12, 2013.

${ }^{68}$ Las dos fases son individualizadas, por ejemplo, por Della Cananea, G., «Italy», en Kassim, H., Peters, B.G. y Wright, V. (eds.), The National Co-ordination of EU Policy. The Domestic Level, cit., p. 107 y ss. Al respecto, se puede hacer referencia también a RonziTTI, N., «European Policy Formulation in the Italian Administrative System», The International Spectator, Vol. 22, n. 4, 1987, p. 207 y ss.; FrANCHINI, C., «L'integrazione europea e il governo delle politiche comunitarie in Italia: organizzazione amministrativa e rapporto con le Comunità Europee», Rivista di diritto europeo, n. 1, 1990, p. 37 y ss.; AA. VV., Indagine conoscitiva sulla partecipazione dell'Italia alle fasi formativa ed applicativa del diritto comunitario, Senato della Repubblica, Roma, 1991. En español, ofrece un marco claro de la situación de las primeras tres décadas CHITI, M.P., «La creación y ejecución del derecho comunitario en los Estados compuestos: Italia», Revista Andaluza de Administración Pública, n. 9, 1992.

${ }^{69}$ Véase al respecto Della Cananea, G., «L'Italia e l'Europa: la politica «comunitaria» nel periodo iniziale del processo di integrazione (1952-1967)», en VOLKMAR HEYEN, E. (ed.), Early European Community Administration - Jahrbüch der Europä̈sches Verwaltungsgeschichte, Nomos, Baden Baden, 1992, p. 207 y ss.

${ }^{70}$ Ley 183/1987: «Coordinamento delle politiche riguardanti l'appartenenza dell'Italia alle Comunità europee ed adeguamento dell'ordinamento interno agli atti normativi comunitari».

${ }^{71}$ Ley 48/1967: «Attribuzioni e ordinamento del Ministero del bilancio e della programmazione economica e istituzione del Comitato dei Ministri per la programmazione economica». 
interna, pero al mismo tiempo se encargaba de promover las actuaciones necesarias para la armonización de la política económica nacional con las políticas económicas de los demás países de las entonces Comunidades Europeas $^{72}$. Aunque a lo largo de los años cambió su composición, se mantuvieron sus «funciones europeas» hasta su traspaso al Comité Interministerial para los Asuntos Comunitarios Europeos (CIACE), cuyo cometido era, por un lado, definir «las líneas políticas del Gobierno en el proceso de formación de la posición italiana en la fase de predisposición de los actos comunitarios y de la Unión Europea» y, por otro lado, asegurar que se cumpliesen las obligaciones derivadas de la UE ${ }^{73}$. Desde el año 2013 se denomina Comité Interministerial para los Asuntos Europeos (CIAE) ${ }^{74}$.

El CIAE es apoyado por un Comité técnico permanente, instituido dentro del Departamento para las Políticas Europeas (el antiguo Departamento para la Coordinación de las Políticas Comunitarias) y compuesto por un representante designado por cada Ministro.

El Departamento se encarga de la coordinación, en la fase ascendente, de las Administraciones estatales competentes, de las Regiones y de los operadores privados para definir la posición italiana a defender en las sedes decisorias europeas, de acuerdo con el Ministro de Asuntos Exteriores. Posteriormente, durante el proceso de toma de decisión en las Instituciones europeas, el Departamento supervisa su evolución para favorecer una adaptación constante de la postura del Estado; finalmente, en la fase descendente, se ocupa de colaborar y monitorizar el procedimiento legislativo de las leyes necesarias para la ejecución o integración de las fuentes comunitarias, y en general de su implementación por parte de los sujetos obligados (especial-

72 Inicialmente estaba formado por el Presidente del Gobierno, el Ministro de Economía, los de Asuntos Exteriores, de Hacienda, de Industria y Comercio, de Agricultura, de Comercio exterior, de participaciones estatales y obras públicas, de Trabajo, Transportes y Turismo (además del Ministro competente para las medidas extraordinarias en el Sur y en las zonas menos desarrolladas del Norte). Participaban en sus reuniones también los demás Ministros competentes según la materia.

${ }^{73}$ Este Comité también se situaba dentro de la Presidencia del Gobierno y en sus sesiones participaban el Presidente, el Ministro para las Políticas Comunitarias, el Ministro de Asuntos Exteriores, el Ministro para los Asuntos Regionales y los demás Ministros competentes en las materias al orden del día.

${ }_{74}$ Véase el art. 2 de la Ley 234/2012, «Norme generali sulla partecipazione dell’'Italia alla formazione e all'attuazione della normativa e delle politiche dell'Unione europea». Lo convoca y preside el Presidente del Gobierno o el Ministro para los Asuntos Europeos si éste le delega. Participan en las sesiones el Ministro de Asuntos Exteriores, el de Economía y Hacienda, el Ministro para los asuntos regionales, turismo y deporte, el Ministro para la cohesión territorial y los otros que tengan competencias relacionadas con los actos en el orden del día. La regulación orgánica anterior se había dado con la Ley 11/2005, «Norme generali sulla partecipazione dell'Italia al processo normativo dell'Unione europea e sulle procedure di esecuzione degli obblighi comunitari». 
mente las Regiones) y de las diferentes Administraciones llamadas a implementar esta regulación ${ }^{75}$.

\section{CONCLUSIONES}

A lo largo del estudio se ha analizado la evolución de los mecanismos de coordinación que se refleja en el formante legal, mientras que no se han investigado los cambios informales en las actuaciones de los diferentes actores. Las líneas generales que la doctrina había evidenciado y que se han descrito con anterioridad se ven sólo parcialmente confirmadas por el análisis jurídico desarrollado en estas páginas.

En primer lugar, el alejamiento de las políticas europeas de la égida del Ministerio de Asuntos Exteriores es cierto sólo en algunos casos: para la «opción cero» y para aquellos sistemas que se han incluido en los modelos 3 y 4, aunque naturalmente este Ministerio sigue siendo competente en relación a la PESC (los Países Bajos se han movido incluso en contra-tendencia, puesto que la coordinación pasó del Ministerio de Economía al de Exteriores).

En segundo lugar, el papel creciente de la Presidencia del Gobierno como sede de la coordinación ${ }^{76}$, si es evidente en los estudios empíricos y cualitativos de las dinámicas concretas, no lo es tanto si nos fijamos en el dato jurídico. Lo que sí parece ser una constante es la importancia del Consejo de Ministros como órgano decisorio final. En tercer lugar, la tendencia a crear sedes de coordinación especializadas tampoco encuentra una confirmación totalmente uniforme en los ordenamientos estudiados.

El único sistema, de los seis examinados, que cumple con estas tres tendencias es el italiano ${ }^{77}$, dado que la estructura del ejecutivo ha sido modificada, por un lado, debido a la creación de un Ministro específico y, por otro lado, se han instituido sedes de coordinación política y administrativa y todas se sitúan en la Presidencia del Gobierno, al estar relacionadas con el Departamento para las Políticas Europeas.

${ }^{75}$ Las Instituciones con las que el Departamento tiene que cooperar son la Conferencia permanente para las relaciones entre Estado, Regiones y Provincias Autónomas de Trento y Bolzano (para toda actividad relacionada con las sesiones comunitarias de la misma); el Parlamento (por ejemplo, la preparación de la documentación y de los informes periódicos) y los demás Ministerios (particularmente el de Asuntos Exteriores y el de Economía y Hacienda). Véase el decreto de organización del Ministro para los Asuntos Europeos del 7 de diciembre de 2012.

${ }^{76}$ Sobre esta línea evolutiva, véase KASSIM, H., «The Europeization of Member State Institutions», Ob. Cit., p. 296.

${ }^{77}$ Se nos permita recordar de nuevo nuestro estudio «Coordinación de las políticas europeas y transformaciones estructurales de los Ejecutivos nacionales (el caso italiano en perspectiva comparada)», Ob. cit., p. 12. 
La falta de convergencia entre los casos de estudio, sin embargo, no es sinónimo de ausencia de una transformación de los sistemas, que han sido todos sometidos a modificaciones desde el comienzo del proceso de integración. A la luz de este dato, merece la pena volver ahora a la pregunta que hemos esbozado en el $\S 2$, es decir, si las reformas están relacionadas con los momentos clave de la evolución de la UE o con coyunturas importantes para el Estado miembro interesado.

La respuesta a esta cuestión no puede sino ser dicotómica. Por una parte, conforme al aumento de las competencias demandadas al nivel europeo, los Ministerios nacionales se han dotado cada vez más de secciones y divisiones especializadas al respecto. Por otra parte, el desarrollo de los órganos de coordinación interministerial parece reflejar causas internas -nacionales-. Lo demuestran las reformas italianas de los años 80 (en una fase en la que se estaba repensando la estructura del Gobierno, que se reformó con la ley 400/1988) o las alemanas de los 60 y 90, dependientes de factores políticos y constitucionales endógenos. Aún más lo prueba la evolución de Bélgica, donde los cambios se han debido a la reforma federal; y parcialmente Francia, donde las funciones de la SGAE representan la transposición del modelo tradicional de coordinación al ámbito europeo. Tampoco la coincidencia de la reorganización italiana y francesa en 2005 parece tener una causa «europea», sino interna. Resulta así que las justificaciones de las reformas de la coordinación en el nivel político más elevado (los Ministerios) -y no meramente administrativo- tienen raíces nacionales y están desvinculadas del desarrollo institucional y normativo de la UE.

Así, gracias a las diferencias persistentes, la observación de este panorama comparativo revela una pluralidad de soluciones potenciales y abre la puerta a vías de circulación, imitación y eventualmente hibridación de los modelos según variables diferentes, desde la forma de gobierno del Estado «importador» o su modelo administrativo hasta la eficacia o el prestigio del sistema. La muestra examinada ofrece ya cuatro posibles combinaciones entre los dos elementos determinantes seleccionados, aunque potencialmente hay más opciones para elaborar fórmulas híbridas.

No son pocos los Estados miembros para los cuales alcanzar una coordinación más eficaz sigue siendo una asignatura pendiente entre las reformas para perfeccionar la participación en los procesos decisorios europeos.

Y España es uno de ellos. Aunque no se puede aquí profundizar en el tema, bastará con recordar que el Informe del Consejo de Estado sobre la inserción del Derecho Europeo en el ordenamiento español de 2008 hizo especial hincapié en este aspecto, propiciando una revalorización del Ministerio de la Presidencia (de cara a un sistema que todavía tiene como protagonista al Ministerio de Asuntos Exteriores y Cooperación, como se vio en el $\S 3)^{78}$. La respuesta a

78 Véase el Informe del Consejo de Estado sobre la inserción del Derecho Europeo en el ordenamiento español de 2008, p. 106 y ss. 
la exigencia de resolver «de forma expeditiva las discrepancias suscitadas una vez intentada la coordinación desde el Ministerio responsable por razón de la materia» podría derivar de una imitación (combinada) de algunas de las soluciones normativas expuestas en el texto, siempre teniendo en cuenta los elementos comunes y diferenciales de los ordenamientos empleados como modelos, para evitar crisis de rechazo. En este caso, el Derecho Comparado cumpliría con una de sus funciones prácticas más acreditadas: el auxilio en el procedimiento de aprobación de actos legislativos.

TITLE: Government coordination of EU policies: a Comparative Legal Analysis of the Founding Members

RESUMEN: El estudio analiza los sistemas de coordinación de las políticas europeas adoptados en los Estados fundadores de la UE. Teniendo en cuenta la evolución normativa, la autora propone una clasificación cuatripartita, basada en la aplicación combinada de distintos elementos determinantes: la existencia o inexistencia de un Ministro para los Asuntos Europeos; la existencia o inexistencia de un órgano de coordinación interministerial específico y su colocación en la Presidencia del Gobierno o en el Ministerio de Asuntos Exteriores. Los resultados del análisis comparado son finalmente cotejados con los de otros estudios anteriores y en las conclusiones se enfoca el tema desde la óptica de la circulación de modelos.

PALABRAS CLAVE: coordinación; Gobierno; politicas europeas; comparación jurídica

ABSTRACT: This study analyses the systems adopted by the founding members of the EU on the coordination of EU policies. Considering their normative evolution, the author proposes a four-way classification based on the combined application of distinct elements: the existence or lack thereof of a Ministry of European Affairs; the existence or lack thereof of a specific organ for inter-ministerial coordination and its situation in the office of the Prime Minister or the Ministry of Foreign Affairs. Finally, the results of the comparative analysis are checked against the findings of previous studies and the conclusions focus on the topic from the perspective of diffusion of models.

KEYWORDS: coordination; Government; EU policies; legal comparison

RECIBIDO: 12.05 .2014

ACEPTADO: 26.05.2014 